\section{DESARROLLO DE VALORES Y ACTITUDES A TRAVÉS DE LA CLASE DE EDUCACIÓN FÍSICA}

\author{
DEVELOPMENT OF VALUES AND ATTITUDES THROUGH PHYSICAL \\ EDUCATION CLASSES
}

\author{
O DESENVOLVIMENTO DE VALORES E ATITUDES POR MEIO DA AULA DE \\ EDUCAÇÃO FÍSICA
}

Didier Fernando Gaviria Cortes*, Francisco Javier Castejón Oliva**
Valores sociales. Actitud.

Estudios de caso.
Palabras clave

Resumen: El presente estudio tuvo como objetivo comprender las actitudes y valores que genera la Educación Física en los estudiantes de secundaria. Para ello se parte de conceptos claves como valores, actitudes y Educación Física. La investigación es cualitativa y se llevó a cabo a través de un diseño de estudio de caso en un instituto de Madrid-España; permitiendo una comprensión de las percepciones, emociones, vivencias y opiniones que tienen los estudiantes sobre los valores y actitudes que se generan en la clase de Educación Física. La información fue recolectada por medio de la observación y del grupo focal. Los resultados muestran, el compañerismo y la cooperación como los valores más importantes dentro de la práctica para los estudiantes; igualmente se evidenciaron actitudes y valores en relación con uno mismo y en relación a la asignatura.

Keywords Social values. Attitude. Case studies.

Palavras-chave Valores sociais. Atitude.

Estudos de caso.
Abstract: The purpose of this study was to understand the attitudes and values promoted by Physical Education in High School students, based on key concepts like values, attitudes and Physical Education. It is a qualitative study conducted through a case study at a secondary education institution in Madrid, Spain. It allowed understanding students' perceptions, emotions, experiences and opinions about the values and attitudes fostered by Physical Education. Data were obtained using observation and a focus group. Companionship and cooperation were found as the most important values for students in the practice. Likewise, results show students' attitudes and values about themselves and the Physical Education class.

Resumo: O presente estudo teve como objetivo compreender as atitudes e os valores gerados pela Educação Física em estudantes do ensino médio. Para isso, partiu-se de conceitoschave como valores, atitudes e Educação Física. A pesquisa é qualitativa e foi realizada através de um projeto de estudo de caso em uma instituição de ensino de Madri, na Espanha. Isto permitiu uma compreensão das percepções, emoções, experiências e opiniões que os alunos têm sobre os valores e atitudes que são gerados na aula de Educação Física. As informações foram coletadas por meio de observação e grupo focal. Os resultados mostram companheirismo e cooperação como os valores mais importantes na prática para os alunos; também atitudes e valores em relação a si mesmos e em relação ao assunto eram evidentes.
*Universidad de Antioquia. Antioquia. Colombia.

E-mail: didier.gaviria@udea.edu.co

** Universidad Autónoma de Madrid. Madrid. España.

E-mail: javier.castejon@uam.es

Recebido em: 12-02-2015 Aprovado em: 05-09-2015 (c) (1) (8) Licence 


\section{INTRODUCCIÓN}

Existen, al menos, dos posibilidades del desarrollo de los valores en educación, por un lado, materias que tengan la exclusividad de incidir en los valores educativos (valores como fin) y que pueden tener diferentes nombres (habitualmente en países laicos, educación para la ciudadanía, educación moral, y en países confesionales puede llegar a dejarse en la religión), quedando el resto de las materias en seguir o mantener cierto acuerdo con esa exclusividad; y por otro lado, se entiende que los valores deben ser tratados en todas las materias por igual (valores como medio), es decir, una transversalidad que alcanza a todas las asignaturas por igual, y que todos y todas deben asumir (BOLÍVAR, 2007, TOURIÑAN, 2006).

Esta tendencia en educación, que toma partido por los valores como medio, entendemos que debe aparecer en las asignaturas, y con ello, también podríamos plantear para la Educación Física (EF), en cuanto que tiene la tarea de formar para el mundo de la vida.

La educación prepara para participar en el mundo en la medida en que proporciona la cultura que compone ese mundo y su historia, transformándola en cultura subjetiva, lo cual presta la forma de presencia nuestra ante los bienes culturales, una manera de ser ante la herencia recibida, a eso llamamos saber. La cualidad de la experiencia cultural de calidad vivida es la preparación más real que puede y debería propiciar la educación. (GIMENO, 2005, p. 35)

En este sentido, la educación en el mundo de la vida consiste en el yo haciendo algo con las cosas del mundo. El ser humano aprende de esta forma a dar importancia a las cosas, estimar y valorar todo aquello que le rodea.

Encontramos que la educación debe tener un cometido de incidir en los valores (es cierto que no solo la educación, por supuesto -véase ORTEGA; MÍNGUEZ, 2003), y que en las asignaturas se debe realizar este desarrollo en valores, en el caso de la EF así debería ser, lo que no sabemos es en qué medida se incide en los valores y cómo se puede llevar a cabo, de la misma forma que también es necesario saber qué entienden y cómo practican esos valores los estudiantes.

\section{ACERCA DE LOS VALORES Y ACTITUDES}

El concepto de valor es un termino polisémico y abarca una gran cantidad de significados que pueden dar lugar a descontextualizar su sentido. La palabra valor se caracteriza por tener múltiples usos en los campos de la vida y la actividad humana (REMOLINA, 2005).

Para Bolívar (1992), los valores son ideales abstractos, que representan las creencias de una persona sobre los modelos ideales de conducta y sobre los fines últimos. Son autoconcepciones que una persona tiene de sí mismo, de los demás y del mundo, y por estos motivos actúa y elige de forma autónoma.

Se entiende que los valores se adquieren a través de los procesos de socialización y de transmisión entre los seres humanos. Es decir, que todas las experiencias de vida que se producen desde la niñez incidirán en el proceso de formación de la personalidad. Se adquiere un estilo de vida por medio de las influencias que le aportan los agentes socializadores como la familia, la escuela, los amigos, los centros deportivos y los medios de comunicación. Es así que el niño y la niña interiorizan los valores, se hace cada vez más evidente con la edad, a través 
de la valoración personal que hace cotidianamente desde sus opiniones, acciones, actitudes y conductas, y de esta forma desarrolla un criterio personal frente a la vida.

Al hablar de valor, es importante tener presente otro concepto como el de actitud, el cual es utilizado en muchas ocasiones en lugar del concepto de valor. Una actitud entonces, "hace referencia a un pensamiento o un sentimiento positivo o negativo que tiene una persona hacia los objetos, las situaciones o hacia otras personas. Está relacionado con los sentimientos y formas de actuar ante hechos o situaciones determinadas" (PRAT; SOLER, 2003, p. 21).

Según Puig (1995, p. 125), "las actitudes son aquellas tendencias o predisposiciones aprendidas y relativamente fijas, que orientan la conducta que previsiblemente se manifestará, ante una situación u objeto determinado". Por lo tanto, pueden ser de carácter positivas 0 negativas según la situación u objeto.

Escámez et al. (2007) dicen que las actitudes son evaluaciones afectivas, pertenecen primordialmente al ámbito de los sentimientos, valoraciones de la realidad que pueden ser perjudiciales o favorables para la vida de las personas, por medio de ellas los sujetos exteriorizan un modo de actuar y definen su posición frente a las personas, las instituciones, las situaciones y las cosas con las que se relacionan cotidianamente.

Desde un punto de vista educativo, hay que tener presente que cuando se desee fomentar, afianzar o modificar determinadas actitudes, se debe de incidir en lo cognitivo, afectivo y conductual. También se debe de tener presente, que la escuela no es la única que influye en el comportamiento de los estudiantes, sino que hay una gran influencia de la familia, los amigos, los medios de comunicación y la sociedad en general en el desarrollo de ellas.

\section{LA EDUCACIÓN FÍSICA COMO POTENCIADORA DE VALORES Y ACTITUDES}

La EF puede generar beneficios en el desarrollo de las personas a nivel psicológico, los estilos de vida, afectivos, sociales y cognitivos, por ello la necesidad de la investigación y evaluación de programas educativos y deportivos que ayuden a comprender la naturaleza de estas contribuciones de la EF (BAILEY, 2006).

En el proceso de esta reflexión han emergido nuevas formas de pensar la $E F$, dando lugar al surgimiento de postulados teóricos que se constituyen en referentes conceptuales, sobre los que se orientan la discusión teórica y las prácticas motrices.

Es así, que Uribe et al. (2003), plantean que es necesario considerar en el concepto de EF una orientación pedagógica que procure e intente el desarrollo de las potencialidades humanas y el trabajo en todas las dimensiones de ser humano.

Para González (1993, p. 49-52), el objeto de la educación es el perfeccionamiento humano, es ayudar a la persona a extraer lo mejor de todas sus capacidad de autonomía y de relación con los demás, y define la EF "como la ciencia y arte de ayudar al individuo en el desarrollo intencional (armonioso, natural y progresivo) de sus facultades de movimiento, y con ellas el resto de sus facultades personales".

La necesidad del desarrollo de valores y aptitudes en la actividad física ha supuesto planteamientos o modelos que han incidido en los mismos, como es el caso de Hellison (1995), con una secuencia de actividades que llegan hasta la responsabilidad personal y la autonomía 
"fuera del gimnasio". En esta misma línea, el trabajo de Escartí (2005) ha mostrado cómo se puede conseguir compromisos personales de actitud siguiendo la línea argumental de Hellison. Por otro lado, Jiménez (2008) ha demostrado cómo se pueden plantear estrategias de intervención para la mejora de los valores en los que la actividad física y el deporte, es un medio propicio para conseguir resultados positivos que permiten vencer las resistencias de los adolescentes.

La EF debe ser algo más que simple actividad física, los medios con los que cuenta (juegos, ejercicios, danzas, deportes, actividades en el medio natural, la expresión corporal), son un potencial que van más allá del desarrollo de las condiciones físicas de las personas, sino que pueden afianzar el carácter; en este sentido, la EF considera las interrelaciones de las dimensiones del ser humano, haciendo un aporte a la formación integral de la persona. Por ejemplo, el trabajo de Fraile (2008), muestra cómo la aplicación de actividades cooperativas en la clase de EF ayuda a solucionar los conflictos y cómo se asumen competencias y responsabilidades por parte del alumnado.

La EF entendida así, como una educación del ser humano que comprende el perfeccionamiento y desarrollo de todas sus dimensiones (cognitiva, afectiva, social, biológica, estética, madurativa, lúdica, erótica, ética, moral, comunicativa, espiritual y motriz), puede convertirse en una gran portadora de desarrollo y calidad de vida de la comunidad educativa, ya que el ser humano no solo se mueve sino que se expresa.

En este sentido, la intencionalidad de esta investigación, estuvo orientada en la reflexión de una EF que trascienda los fines y objetivos de aprendizaje centrados en el desarrollo de habilidades motrices y capacidades físicas, hacia una EF que busque la formación de valores y actitudes en la persona, una educación para la vida. Es así, que nos preguntamos sobre ¿Cuáles son las actitudes y valores que se generan en los estudiantes de secundaria a través de las clases de EF?

\section{METODOLOGÍA}

El desarrollo de ésta investigación se llevó a cabo a través de un estudio cualitativo, puesto que es a partir de la metodología cualitativa que se puede abordar según Galeano (2004a), la realidad subjetiva e intersubjetiva como objetos legítimos de conocimientos científicos, buscando desde la interioridad de los actores sociales las lógicas de pensamiento que guían las acciones sociales. Se puede comprender la realidad en su contexto natural, tal y como sucede, intentando sacar sentido o interpretar los fenómenos de acuerdo con los significados que tienen para las personas implicadas en la clase de EF y las lógicas de pensamiento que guían sus acciones (RODRÍGUEZ; GIL; GARCÍA, 1999). Taylor y Bogdan (1996) argumentan, que si estudiamos a las personas cualitativamente llegamos a conocerlas en lo personal y a experimentar lo que ellas sienten en sus luchas cotidianas en la sociedad.

Para la investigación se eligió como diseño el estudio de caso puesto que según Stake (1998), es algo específico y complejo que se encuentra en funcionamiento y de lo cual se pretende lograr una mayor comprensión y profundizar en aspectos que no son habituales mediante otros diseños. El caso puede ser una persona, un programa, un grupo social; el cual puede apreciarse en su singularidad y complejidad al insertarse en sus contextos y su interrelación con ellos. 
Según Galeano (2004b), el estudio de caso le permite al investigador alcanzar mayor comprensión y claridad sobre un tema o aspecto teórico concreto, o indagar un fenómeno, una población o una condición en particular; estos últimos aspectos, fueron precisamente los que esta investigación pretendió desarrollar en los estudiantes de la ESO (Educación Secundaria Obligatoria).

La población estudiada correspondió a los estudiantes de cuarto curso de secundaria de un instituto de la comunidad de Madrid; por ser personas que poseen una experiencia y conocimiento en cuanto a la asignatura a lo largo de su proceso escolar, se encontraban entre los 15 y 17 años (Quadra 1).

\begin{tabular}{|c|c|}
\multicolumn{2}{c}{ Quadro 1 - Población } \\
\hline Sexo & Edad \\
\hline 41 Chicos & Entre 15 y 17 años de edad \\
40 Chicas & Entre 15 y 17 años de edad \\
\hline
\end{tabular}

Quadro 2 - Clases de EF y contenidos

\begin{tabular}{|c|c|}
\hline Sesiones de EF & Contenidos \\
\hline 21 sesiones observadas & 3 Contenidos de EF desarrollados \\
\hline \multicolumn{2}{|c|}{ Fuente: Autor }
\end{tabular}

Las clases de EF se desarrollan dos veces por semana, en sesiones de 50 minutos; los contenidos trabajados en las clases observadas fueron los deportes colectivos, la expresión corporal y condición física (Quadro 2).

\section{ESTRATEGIAS DE RECOLECCIÓN DE INFORMACIÓN Y ANALISIS DE LOS DATOS}

Dada la naturaleza del fenómeno a estudiar, las técnicas que se usaron para la recolección de la información fueron la observación y el grupo focal. En particular se realizó la observación participante, haciendo la salvedad de que esta observación se hizo con el mínimo de intervención; a partir de estas estrategias se pudo identificar los valores y actitudes potenciados por la práctica de la EF en los estudiantes, y reconocer además sus percepciones, sentimientos y preocupaciones frente a la incidencia de las prácticas (O.C.n, hace referencia a la observación realizada en la presentación de los resultados y G.F.n, corresponde a grupo focal).

Basados en la propuesta de Taylor y Bogdan (1996), el análisis de la información se llevó a cabo siguiendo un proceso que consta de tres fases: fase de descubrimiento, fase de codificación y fase de relativización de los datos.

\section{RESULTADOS, ANÁLISIS Y DISCUSIÓN}

\subsection{Concepciones sobre valores y actitudes de los estudiantes}

Se puede decir, que los valores personales dependen en gran medida de los factores sociales, y del sistema de valores que posee e impone a sus miembros (BOLÍVAR, 1998). En este sentido los estudiantes nos dijeron: 
"un valor es algo que hay que acatar para el bien común de todos y la diversión de todos" G.F.3.

"algo que tu piensas que esta bien sabes que esta bien... como una pauta, sí una pauta que tienes que seguir común, bueno a veces no común, pero generalmente debe de haber comunes para que podamos regular un poco la convivencia" G.F.6.

Podemos ver como los conceptos desarrollados por los estudiantes tienen una aplicación a la vida real, se puede decir, que el comportamiento de las personas, se orienta a partir de unos valores que cada sujeto ha desarrollado, que los valores son compartidos y aceptados por los integrantes de una sociedad (ESCARTí et al., 2005).

Las actitudes en cambio son evaluaciones afectivas, que pertenecen primordialmente al ámbito de los sentimientos, valoraciones de las realidades como perjudiciales o favorables para la vida de las personas, es a través de ellas que las personas manifiestan un modo de comprender y definir su posición frente a otras personas, las instituciones, las cosas con las que se relaciona y las situaciones que vive día a día (ESCÁMEZ et al., 2005). Al respecto plantearon los estudiantes:

"una actitud es la forma de comportarnos... la forma de cumplir esos valores" G.F.5. "una forma de ser, aplicar los valores, claro, de comportarse de una manera, tener un mínimo de respeto" G.F.6.

Se puede ver como las actitudes están relacionadas directa o indirectamente con objetos o situaciones específicas, es decir, simplemente señala la disposición favorable 0 desfavorable de las personas hacia algo en unas circunstancias concretas. En este caso con relación a la gente, al juego y con uno mismo.

\section{2 ¿Qué contenidos actitudinales se generan en la clase de Educación Física?}

Teniendo en cuenta la propuesta de Prat (2001) se agruparon los principales contenidos actitudinales que se trabajan en EF en cuatro grandes bloques: actitudes y valores en relación a uno mismo, en relación a los demás, en relación a las normas y en relación a la materia.

Los tres primeros bloques corresponden a un análisis e interpretación más objetiva (eje filosófico-moral) "hace referencia a lo que -por razones justificables- merece ser apreciado, como formas deseables de comportamiento o vida" (BOLÍVAR, 1998, p. 19) y el último bloque tiene una perspectiva más subjetiva (eje más psicológico), como la valoración de la materia, el interés del estudiante hacia la EF. Para nuestro interés en concreto, la respuesta que nos da un estudiante en relación a la clase de EF es también un compendio de lo que conoce o piensa acerca de ello, de qué manera lo percibe, y su forma de actuar en relación con ello.

Teniendo en cuenta lo anterior, se encontró en el estudio que los contenidos que aparecen con más frecuencia en la clase de EF son los que tienen que ver con actitudes y valores en relación a los demás y el bloque de actitudes y valores en relación a uno mismo.

\subsubsection{Valores y actitudes en relación con los demás}

Este contenido en la clase de EF se refiere a esas relaciones que se dan entre estudiantes y con el profesorado. Estos valores dotan los aprendizajes de una mayor funcionalidad y aplicación con la realidad que vive el estudiante como sujeto social. 
En este sentido se identificó que en las clases de EF del instituto sobresalen los valores de cooperación, compañerismo, el conflicto y la competición. Estás dos ultimas también tienen aspectos de carácter no deseado o negativo en la formación del alumnado, la igualdad de las mujeres en algunas actividades y su participación en ellas es otro punto negativo a la hora de reflexionar y comprender lo que se genera en la práctica.

\subsubsection{La cooperación}

Para Fernández (2009, p. 76) "cooperar podría traducirse por ayudar conjuntamente con, ayudarse, apoyarse mutuamente, interesarse uno por el otro". Plantea que la cooperación añade a la colaboración un plus de solidaridad, de ayuda mutua o de generosidad que hace que aquellos que simplemente estén colaborando, sean más eficaces en el trabajo que deben realizar, además de crear lazos afectivos más profundos debido a ese objetivo común a alcanzar por todos los participantes. En este sentido se observó y nos dijeron los estudiantes:

"haces más grupo, que el grupo funcione mejor, porque trabajamos en equipo... pues nos relacionamos mejor entre todos los de la clase, así aprendes a relacionarte con tu equipo" G.F.1.

"a veces que lo toque es más bonito, una jugada tocándolo todos y aunque falles, aunque llegue a medio campo, queda más bonito, que sea solidario... a que diga: mira que bien juego a hockey porque soy el mejor y se jugar individual" G.F.4.

"el profesor les explica y luego los alumnos y alumnas se organizan en el espacio y comienzan a desarrollar las actividades planteadas en la planilla; los equipos se corrigen y se ayudan entre ellos, se motivan para que las cosas les salga bien a todos los integrantes del equipo" O.C.6

Como se puede notar, los estudiantes participan en el desarrollo de los juegos, se involucran en su aprendizaje, resuelven problemas entre todos los participantes de la clase, se escuchan, comparten ideas, se preocupan por el otro; siendo todo este tipo de actividades habituales en la vida cotidiana, de la misma forma que comentaron Fraile (2008) y López y Eberle (2003). De allí la importancia que los estudiantes comprendan su valor y lo implementen en su vida en sociedad.

\subsubsection{El compañerismo - amistad}

Para algunos autores, uno de los problemas de la vida en sociedad es la falta de amistad, ya que si no tenemos amigos, es que ha desaparecido la confianza en las relaciones, no se dedica tiempo al cultivo de la misma, no se escucha al otro sin interés y de forma paciente, y no se demuestra afecto y confianza cuando un amigo pasa alguna dificultad ( $\mathrm{POCH}, 2008)$. Es así que se observó y los estudiantes nos dicen que aprenden en la clase:

\footnotetext{
"estar en grupo... la confianza en otros de tu equipo, en hacerte más amigo de otros compañeros" G.F.1.

"también intentan por así decirlo socialicemos, que no estemos con las mismas personas siempre y que trabajemos con otras personas... el compañerismo, o sea a trabajar en equipo y pasárselo bien... bueno también a resolver situaciones o si un compañero sabe hacer algo, le cuesta más pues ayudarle" G.F.6.

"entre todos deciden jugar "te pillo" en cadeneta, algunos se proponen para iniciar cogiendo a los demás compañeros, los alumnos se divierten y se ríen durante el juego, conversan, hacen bromas a la vez juegan todos, parece que se disfruta de este momento" O.C.3.
} 
La conducta entre los estudiantes presenta una dimensión de reciprocidad en cuanto conductas deseables por las personas (JIMÉNEZ, 2008, HELLISON, 2003), por ejemplo: compartir las cosas y las actividades, ayudar al otro cuando lo necesita, dar confianza a los demás; aspectos que se pueden identificar en los testimonios de los estudiantes, además se puede hablar de tener en cuenta los gustos, preferencias y sentimientos de las otras personas.

\subsubsection{Competición}

Las relaciones humanas que se presentan en la práctica de la actividad física, el deporte y la $E F$, son en ocasiones enriquecedoras, aunque en otras ocasiones propician sentimientos negativos y opiniones que alejan al área de ser un lugar de encuentro y desarrollo humano. En este sentido los estudiantes plantearon:

"todo el mundo quiere ganarle al otro y eso, se esfuerzan más" G.F.1.

"es buena la competitividad si sabes modelarla, porque sino tienes competitividad no te esfuerzas, no haces nada por hacerlo divertido o interesante... el deporte" G.F.3.

"es buena porque hace que al ver que tienes otro que esta adelante haces superarle a él y así, te esfuerzas, sucesivamente hasta poder dar más" G.F.5.

Y es que la competencia puede por una parte formar al estudiante para una vida en sociedad, por medio de valores como el esfuerzo, la superación, el respeto a las normas y a los compañeros de juego, tolerancia, etc. Sin embargo también puede formar para la exclusión, el individualismo, el menosprecio, la desigualdad, etc. De allí que en el análisis de la información se encuentran algunos planteamientos en ambos sentidos.

"durante el juego un alumno y una alumna se chocaron corriendo por cumplir el objetivo del juego, por lo cual se genero una discusión bastante fuerte entre ellos, cruzando palabras y miradas ofensivas, los demás compañeros observan lo sucedido sin decir nada al respecto" O.C.3.

"a veces se te pueden ir los nervios, pero siempre los demás compañeros intentan que te calmes y que hables las cosas... si que no acabes pegándole a nadie" G.F.6.

Otra de las críticas que se hacen a la competición, tiene que ver con la transmisión de valores dominantes actualmente en la sociedad como el individualismo, el triunfo, éxito particular, entre otros. Un estudiante nos dijo:

"hombre al que sabe jugar hay que pasársela,... uno no sabe jugar no se la voy a pasar a esa persona, se la pasas a una que sabe jugar" G.F.5.

La competición o más específicamente el deporte de competición, también ha sido cuestionado en relación con los aspectos relativos al género... algunos valores asociados a ella como energía, habilidad, valor, fuerza, decisión, agresividad... han estado tradicionalmente vinculados a la actividad masculina (CASTEJÓN, 2010, VELÁSQUEZ, 2004). Mientras la motricidad femenina está relacionada con otro modelo vinculado a las actividades rítmicas y expresivas y relacionado con valores como la sumisión, la cooperación y la suavidad y gracia en el movimiento (RUíZ, 2004).

"a mi me pasa mucho cuando no se hacer algo, pues empiezan todos que no sabes hacerlo, pues no lo hagas o cuando estamos jugando en equipo me dicen, tu quédate aquí que nosotros vamos y metemos el gol por ejemplo... normalmente siempre son los chicos, hay un machismo increíble; como que las chicas no pueden 
jugar al fútbol ni al hockey, ni nada por que no saben. Algunas personas si hacen eso, que no dejan jugar a las chicas porque no se les da bien ese deporte... o muchas veces te lo echan y dicen ¿vez, vez? Como vosotras se tienen que dedicar más a este y menos a fútbol ¿sabes?” G.F.3.

"normalmente los tíos como ven un balón ya son los dueños del balón y las chicas fuera, eso no me mola ¿sabe?, porque también las chicas sabemos jugar, no solamente los tíos... muchos chicos que van a equipos de fútbol por las tardes, pues claro saben que juegan bien y se pasan la pelota entre ellos y nosotras nos quedamos como de... nosotras no tocáis el balón; y si no nos ponen de porterías" G.F.5.

Teniendo en cuenta lo anterior, se puede decir, que la competición se puede constituir en un buen medio en la educación de valores, y dirigida por caminos erróneos, puede convertirse en la antítesis de los instrumentos adecuados para la formación en principios éticos (RUíZ, 2004).

\subsubsection{El conflicto}

Hablar de conflicto es hablar de naturaleza humana, pues donde existen relaciones humanas se hace evidente el conflicto. Los seres humanos reunidos en colectivo viven múltiples y variadas experiencias con otras personas en diversos espacios y escenarios, lo cual los hace susceptibles de experimentarlo en muchos momentos de su existencia (ARBOLEDA; GAVIRIA, 2009).

Desde una mirada diferente el conflicto es visto como positivo y creador, como un valor y una actitud, en el cual aquellas personas o grupos inmersos en él tienen la oportunidad de transformarse y desarrollarse como sujetos o colectivos humanos (JARES, 2002). En las clases de EF del instituto, los actores están representados por el profesor y los estudiantes, el profesor en este caso hace de intermediario para facilitar la resolución de conflictos en las clases, mostrando interés en escuchar a las partes involucradas en los conflictos presentados durante las sesiones de clase, con el ánimo de conocer las causas del conflicto y contribuir en la solución a dichas situaciones.

\footnotetext{
"si ve que es muy grave, que van a pegarse o cualquier cosa, pues ya interviene, sino nos deja que nosotras resolvamos la situación a ver como lo hacemos, intenta que aprendamos a resolver esas situaciones sin llegar a las manos" G.F.6.

"depende si es una broma, si es una broma y va a más pues al final la arreglas entre los que hacen la broma, pero si va a más el profesor se para, habla con cada uno" G.F.2.

"la profesora termina la clase y llama a todo el grupo para hablar acerca de lo que esta sucediendo con los dos compañeros agresivos" O.C.7.
}

Se puede decir que el profesor entiende el conflicto como algo positivo del cual se puede generar aprendizajes (FRAILE, 2008, LÓPEZ; EBERLE, 2003), asumiendo el diálogo como la primeria estrategia en el proceso de negociación, conciliación y toma de decisiones con participación de los estudiantes; afianzando así valores en los estudiantes.

\subsection{Actitudes y valores en relación a uno mismo}

Resulta importante este apartado, ya que configura las estructuras o las bases personales básicas, sobre las que se construye cualquier posibilidad de progresar en el aprendizaje y en la formación del sujeto. Se trata de actitudes muy básicas que afectan de una 
manera fundamental todo el proceso escolar de los estudiantes; son prerrequisitos para las otras actitudes y valores a desarrollar dentro del contexto escolar (TRILLO; RUBAL; ZABALZA, 2003). Se podría ubicar aquí, la seguridad en sí mismo, la autoestima, el autoconcepto, el deseo de aprender junto a una actitud positiva hacia la escuela y lo escolar.

Para este caso en particular, se identificó que la clase de EF fortalece actitudes y valores como desinhibición y la relajación en los estudiantes.

"también para relajarse y eso... porque estas agobiado con los exámenes y haces la Educación Física y como que te desconectas de todo" G.F.3.

"esta hora es como para que te despejes un poco de las otras clases... de airarse un poco de las clases... es como relajarte, para despejarte y un poco de esfuerzo... yo creo que eso es lo bueno, para estar con los amigos" G.F.7.

Los testimonios muestran como la clase genera en los estudiantes actitudes de relajación, desinhibición, superación; se trata de pasarlo bien; hacen referencia al estado que experimentan cuando se realiza EF. Se percibe la clase y los contenidos que en ella se utilizan (DEVÍS; PEIRÓ, 2011) como un espacio catártico en el que se sienten inmersos cuando hacen EF, la clase produce un estado de bienestar y una sensación de gratificación intrínseca; lleva consigo una motivación y gratificación interna que permite a los estudiantes un rato de esparcimiento dentro de la institución educativa.

\section{CONCLUSIONES}

Los contenidos actitudinales que aparecen o se generan en los estudiantes con más frecuencia en la clase de EF son: la cooperación y el compañerismo, son los más significativos para los estudiantes. También se presenta la competencia como una actitud buena, en el sentido que invita al esfuerzo y la diversión, mientras otros estudiantes la ven de forma negativa al ser excluyente con las mujeres y en muchas ocasiones provocar agresividad en los estudiantes. El conflicto emerge como medio para el diálogo entre los protagonistas de la clase o como medio de disciplina y control del profesor.

Se ve entonces la necesidad de plantear alternativas en el proyecto curricular, que lo lleve a ser más equilibrado en el conjunto o bloques de actividades de competición, donde se contemplen otras expresiones motrices de carácter más femenino y donde los intereses, gustos y competencias de las chicas sean resaltadas por el grupo en general.

La clase de EF es considerada por los estudiantes como un mecanismo de motivación o interés para crear estilos de vida saludable, pudiéndose formar actitudes para la vida adulta, ya que es el único momento en que independiente de su capacidad atlética, tienen la oportunidad de participar en actividades físicas o deportivas.

La clase de EF es un espacio donde se pretende la formación integral del ser humano, se develan procesos de socialización y buenas relaciones entre los actores (estudiantesprofesor), esto facilita que en el proceso el profesor sea consiente de una intencionalidad por el desarrollo de valores y actitudes positivas en los estudiantes.

Para los estudiantes la clase de EF genera en ellos una actitud de relajación y desinhibición, se trata de pasarlo bien mientras estas en la clase y sienten la asignatura como un momento de esparcimiento dentro del horario escolar, des-estrés, de descanso de las otras asignaturas. 
Se pone de manifiesto las posibilidades de la EF como medio para el desarrollo de valores, si se logra la sinergia entre todos los componentes del currículo y del proceso de enseñanza y aprendizaje, pero esto debe de estar intencionado y de ser coherente con la filosofía o ideología del proyecto educativo.

\section{REFERENCIAS}

ARBOLEDA, Victor; GAVIRIA, Didier. El conflicto: aprendizaje para la convivencia o escenario para ejercer el poder de excluir como castigo. El caso de la Escuela Popular del Deporte en el corregimiento de San Cristóbal, Medellín, 2007-2008. Revista Educación Física y Deporte, Medellín, v. 28, n. 1, p. 29-38, 2009.

BAILEY, Richard. Physical education and sport in schools: a review of benefits and outcomes. Journal of School Health, Malden, v. 76, n. 8, p. 397-401, 2006.

BOLÍVAR, Antonio. Los contenidos actitudinales en el currículo de la reforma: problemas y propuestas. Madrid: Escuela Española, 1992.

BOLÍVAR, Antonio. Educar en valores una educación de la ciudadanía. Andalucía: Junta de Andalucía-Consejería de Educación y Ciencia, 1998.

BOLÍVAR, Antonio. Educación para la ciudadanía: algo más que una signatura. Barcelona: Graó, 2007. CASTEJÓN, Francisco. Deporte como concepto y aplicación. In: CASTEJÓN, Francisco. Deporte y enseñanza comprensiva. Sevilla: Wanceulen, 2010. p. 11-34.

DEVÍS, José; PEIRÓ, Carmen. Sobre el valor educativo de los contenidos de la educación física. Tándem, Barcelona, v. 35, p. 68-74, 2011.

ESCÁMEZ, Juan et al. El aprendizaje de valores y actitudes: teoría y práctica. Barcelona: Octaedro, 2007.

ESCARTÍ, Amparo. El programa de responsabilidad personal y social: aspectos conceptuales y metodológicos. In: ESCARTÍ, Amparo et al. Responsabilidad personal y social a través de la educación física y el deporte. Barcelona: Graó, 2005. p. 29-50.

FERNÁNDEZ, Javier. El modelo comprensivo (Teaching Games for Understanding) TGfU. In: Méndez, Antonio. Modelos actuales de iniciación deportiva unidades didácticas sobre deportes de invasión. Sevilla: Wanceulen. 2009. p. 75-99.

FRAILE, Antonio. La resolución de conflictos en y a través de la educación física. Barcelona: Graó, 2008.

GALEANO, María. Diseño de proyectos en la investigación cualitativa. Medellín: Universidad Eafit, 2004a.

GALEANO, María. Estrategias de investigación social cualitativa: el giro en la mirada. Medellín: La Carreta, 2004b.

GIMENO, José. La educación que tenemos, la educación que queremos. In: IMBERNÓN, Francisco. La educación en el siglo XXI: los retos del futuro inmediato. Barcelona: Graó, 2005. p. 29-52.

GONZÁLEZ, Manolo. La educación física: fundamentación teórica y metodológica. In: BLÁZQUEZ, Domingo. Fundamentos de educación física para la enseñanza primaria. Barcelona: Inde, 1993. p. 53-57.

GUTIÉRREZ, Melchor. Valores sociales y deporte: la actividad física y el deporte como transmisores de valores sociales y personales. Madrid: Gymnos, 1995. 
GUTIÉRREZ, Melchor. Manual sobre valores en educación física y el deporte. Barcelona: Paidós, 2003.

HELLISON, Donald. Teaching responsibility through physical activity. Champaign: Human kinetics, 2003.

JARES, Xesús. La educación para la convivencia como proceso de alfabetización en conflictos.

Revista Interuniversitaria de Formación del Profesorado, Zaragoza, v. 44, p. 79-92, 2002.

Disponible en: <http://dialnet.unirioja.es/servlet/articulo?codigo=249633>. Acceso en: 10 mayo 2012.

JIMÉNEZ, Pedro. Manual de estrategias de intervención en actividad física, deporte y valores. Madrid: Síntesis, 2008.

LÓPEZ, Víctor; EBERLE, Thomas. Utilizar juegos para aprender a resolver conflictos. Tándem, Barcelona, n. 10, p. 41-51, 2003.

ORTEGA, Pedro; MÍNGUEZ, Ramón. Familia y transmisión de valores. Teoría de la Educación. Revista Interuniversitaria, Salamanca, n. 15, p. 33-56, 2003.

PRAT, María. Actitudes, valores y normas en educación física: reflexiones, problemáticas y propuestas para su integración en la escuela. Tándem, Barcelona, n. 2, p. 7-20, 2001.

PRAT, María; SOLER, Susana. Actitudes, valores y normas en la educación física y el deporte: reflexiones y propuestas didácticas. Barcelona: Inde, 2003.

POCH, Concepció. La amistad: aula de innovación educativa. Barcelona, jun. 2008. Disponible en: $<$ http://aula.grao.com/revistas/aula/172-etica-y-educacion/la-amistad $>$. Acceso en: 5 jun. 2012.

PUIG, Joseph. La educación moral en la enseñanza obligatoria. Barcelona: Institut de Ciéncies de I'Educació Universitat de Barcelona-Horsori, 1995.

REMOLINA, Gerardo. La formación en valores. Bogotá, abr. 2005. Disponible en: $<\underline{h t p: / /}$ sistemaucem.edu.mx/bibliotecavirtual/oferta/licenciaturas/contaduria/LCD321/formacion en valores pdf.pdf>. Acceso en: 3 jun. 2012.

RODRÍGUEZ, Gregorio; Gil, Javier; García, Eduardo. Metodología de la investigación cualitativa. Málaga: Aljibe, 1999.

RUÍZ, Jesús. Las actividades físicas cooperativas: una oportunidad para ampliar el valor educativo de nuestra área curricular. Barcelona, ene. 2004. Disponible en: http://www.grao.com/revistas/ tandem/014-juegos-cooperativos/las-actividades-fisicas-cooperativas-una-oportunidad-para-ampliar-elvalor-educativo-de-nuestra-area-curricular. Acceso en: 24 may. 2012

STAKE, Robert. Investigación con estudio de casos. Madrid: Morata, 1998.

TAYLOR, Steven; BOGDAN, Robert. Introducción a los métodos cualitativos de investigación. Barcelona: Paidós, 1996.

TOURIÑAN, José. Educación en valores y experiencia axiológica: el sentido patrimonial de la educación. Revista Española de Pedagogía, La Rioja, n. 234, p. 227-248, 2006.

TRILLO, Felipe; RUBAL, Xosé; ZABALZA, Miguel. La educación en actitudes y valores: dilemas para su enseñanza y evaluación, Rosario: Homo Sapiens, 2003.

URIBE, Iván et al. Pedagogía de la motricidad como estrategia de promoción de la salud. Revista Educación Física y Deporte, Medellín, v. 22, n. 1, p. 59-71, 2003.

VELÁZQUEZ, Roberto. Deporte y educación física: la necesidad de una reconceptualización de la enseñanza deportiva escolar (de un 'deporte educativo' a una 'educación deportiva'). In: LÓPEZ, Víctor; Monjas, Roberto; Fraile, Antonio. Los últimos diez años de la Educación Física escolar. Valladolid: Universidad de Valladolid, 2004. p. 55-76. 